Working Papers

\title{
Institute of
}

Mathematical

Economics

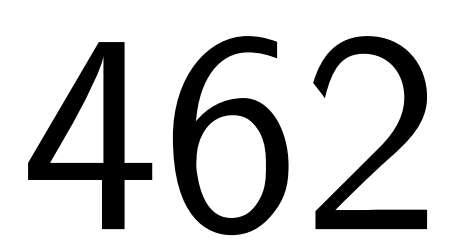

March 2012

\section{Inferring preferences from choices under uncertainty}

Christoph Kuzmics

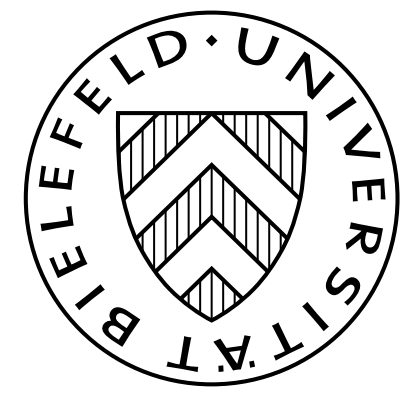




\title{
Inferring preferences from choices under uncertainty
}

\author{
Christoph Kuzmics*
}

March 7, 2012

\begin{abstract}
If a decision maker, in a world of uncertainty a la Anscombe and Aumann (1963), can choose acts according to some objective probability distribution (by throwing dice for instance) from any given set of acts, then there is no set of acts that allows an experimenter to test more than the Axiom of EUOL (that the DM evaluates objective lotteries with an expected utility function). In fact there is no (common) experimental design that allows an experimenter to test more than EUOL. For any decision problem (or set of decision problems), for any preference relation that satisfies the Axiom EUOL, and for any optimal choice (or collection of choices) given this preference relation, there is another preference relation that satisfies EUOL plus the Savage axioms, for which this choice is also optimal.
\end{abstract}

Keywords: ambiguity, decision theory, Knightian uncertainty, experiments JEL codes: C72, C81, C90, D01, D03, D81

*Institute of Mathematical Economics, Bielefeld University, christoph.kuzmics@uni-bielefeld.de. I would like to thank Herbert Dawid, Christoph Diehl, Chiaki Hara, and Frank Riedel for helpful comments and suggestions. Additional comments welcome! 


\section{Introduction}

Suppose a decision maker (DM) is asked to make choices in world in which there is, in addition to possibly risk, also Knightian uncertainty ${ }_{1}^{1}$ Risk is understood to mean that there is an objective and known probability distribution which describes it. Uncertainty is such that there is a set of states of nature, and nature will ultimately choose one state to realize, but there is no known objective probability distribution with which nature does this.

The decision maker is asked to choose from the set of all acts. An act, as defined by Anscombe and Aumann (1963), is a function that attaches objective probability distributions over monetary outcomes to every state of nature.

Savage (1954) has provided axioms a preference relation over acts should satisfy such that a DM with such a preference relation behaves as if she is guided in her decisions by two objects. One, she holds an expected utility function in the sense of von Neumann and Morgenstern (1944). Two, she holds a unique subjective belief, which is a distribution over the states of nature, with which she evaluates all acts.

It is well known that not all individuals behave as if they have expected utility functions for objective lotteries, since at least Allais (1953). Supposing individuals do behave as if they have expected utility functions for objective lotteries Ellsberg's (1961) experiments were designed to test whether individuals' choices are consistent with Savage's (1954) axioms ${ }^{2}$ In this paper I ask whether it is at all possible to design an experiment that can be used to differentiate between preferences that satisfy only the axiom that objective lotteries are evaluated according to some expected utility function (Axiom Expected Utility of Objective Lotteries; EUOL) and preferences satisfying this axiom plus the Savage axioms.$^{3}$ My answer is that it is not possible if two things are true.

First, the DM when given a decision problem, a set of acts, is allowed to choose an act randomly ${ }^{4}$ To put this in the language of game theory, I call the set of acts, the decision maker has to choose from, her set of pure strategies. I find it difficult to deny the DM to choose from the bigger set of mixed strategies, i.e. the set of objective probability distribution over the set of acts given to her. This assumption, if one can call it that, seems very uncontroversial to me. After all, we always allow this in the analysis of games, such as matching pennies, for instance. I simply "extend" it, following Raiffa (1961) to one-player games.

Second, if I allow the DM to choose random acts, I must complete the DM's preference to cover the space of not only all acts, but also all random acts. The only rational way to do this, in my opinion, I provide as an axiom, which I call the Axiom of Point-Wise

\footnotetext{
${ }^{1}$ This is the distinction made by Knight (1921).

${ }^{2}$ Such as those experiments surveyed in Camerer and Weber (1992). The evidence is interpreted to indicate that individuals are uncertainty (or ambiguity) averse.

${ }^{3}$ Alternative preference relations to Savage's that have been proposed include Gilboa and Schmeidler (1989), Schmeidler (1989), Klibanoff, Marinacci, and Mukerji (2005), and Maccheroni, Marinacci, and Rustichini (2006).

${ }^{4}$ This idea was first proposed by Raiffa (1961). In fact, this paper can be understood of a formalization of the full consequences of Raiffa's (1961) argument.
} 
Understanding of (Objective) Mixtures over Acts (Axiom PUMA). It says that the DM applies the randomization over acts in every state. Thus, the DM generates for every state a new objective lottery, the combination of the objective lotteries given by the various acts and the objective probability distribution over acts. This gives rise to a new act in the space of all acts (not all random acts). The Axiom PUMA then finally says that the DM considers the random act and the such constructed new act as identical (she is indifferent between the two). This completes the description of the DM's preferences over all random acts.

In other words, the DM faces three kinds of uncertainty. Once states realize she faces known objective randomness. States are realized subject to unknown (possibly subjective) uncertainty. Acts are chosen by the DM herself according to some, obviously objectively known randomness. The Axiom PUMA simply says that the two sources of objective uncertainty can be combined to provide a single objective uncertainty (for every state). In fact if one accepts (as one must as far as I can see) that both sources of objective randomness are of the same "order", the axiom PUMA really follows from EUOL (by force of the reduction of compound lotteries).

The main result of this paper is then as follows. For any preference relation that satisfies Axioms EUOL and PUMA and any given single decision problem (a set of acts) and any optimal random choice from this decision problem (given the preference) there is another preference relation satisfying the Savage axioms for which the given choice is also optimal. Thus, there is no single decision problem that allows an observer to conclude that the DM's preferences are not consistent with the Savage axioms (under the assumption that they are consistent with von-Neumann Morgenstern axioms for objective lotteries). Of course, that individuals' preferences do not necessarily satisfy the vonNeumann Morgenstern axioms is well known since at least Allais (1953). I then also argue that there is also no set of decision problems which could be used to differentiate Savage from non-Savage preferences, under the assumption of EUOL.

The paper proceeds as follows. Section 2 provides a single example, a small variation of Ellsberg's (1961) experimental design, which is thoroughly discussed to demonstrate the main theorem of the paper as well as the key Axiom of Point-Wise Understanding of (Objective) Mixtures over Acts (PUMA). It has four subsections, each dedicated to a class of experimental designs. In Subsection 2.1 the DM is given a single decision problem. In Subsection 2.2 the DM is given a set of decision problems, with the understanding that one will be objectively randomly chosen to be used to pay the DM. In Subsection 2.3 the DM is again given a set of decision problems, with the understanding now that the DM is paid the sum of all the consequences of her choices. In Subsection 2.4 the DM is allowed to hold beliefs over the correlation of the state of nature and the sequences of decision problems given to her. The argument given there is known and has been discussed extensively already by Shmaya and Yariv (2008), see also Al-Najjar and Weinstein (2011). Section 3 then provides the formal setup. Sections 4 provides the main theorem in full generality for a single decision problem (generalizing Subsection 2.1). Sections 5 and 6 (generalizing Subsections 2.2 and 2.3) discuss the cases in which the DM is given multiple decision problems. 


\section{A motivating example}

In this section I will discuss as my prime example a slight variation of Ellsberg's 2-color urn problem. The variation is there to avoid having to deal with indifferences, and, thus, to make the explanation simpler. All results of this paper are illustrated using this example and then proven in full generality in the main body of this paper.

There are two urns, a risky and an ambiguous one. The risky urn contains 100 balls, of which 49 are red, 49 are white, and 2 are blue. The ambiguous urn contains 100 balls, all of which are white or red. The exact composition of red and white balls in the ambiguous urn is not known.

The decision maker (DM) will have to make choices from subsets of the sets of all acts. While acts are defined in Section 3, in this section it suffices to consider only the following three acts. Act $f_{0}$ pays out 1 monetary unit if we draw a red ball from the risky urn and pays out 0 otherwise. This is, therefore, a constant act, as its payout does not depend on the composition of the ambiguous urn. Act $f_{1}$ pays out 1 monetary unit if we draw a red ball from the ambiguous urn and pays out 0 otherwise. Act $f_{2}$ pays out 1 monetary unit if we draw a white ball from the ambiguous urn and pays out 0 otherwise.

It is useful and customary to model this situation as there being a state space $\Omega=\{R, W\}$, the state being the color of the ball which was randomly drawn from the ambiguous urn. For each state we record the objective probability of winning 1 monetary unit. Note that the outcome space is simply $Y=\{0,1\}$. The three acts can thus be summarized in the following table.

\begin{tabular}{c|ccc} 
state & $f_{0}$ & $f_{1}$ & $f_{2}$ \\
\hline$R$ & $\frac{49}{100}$ & 1 & 0 \\
$W$ & $\frac{49}{100}$ & 0 & 1
\end{tabular}

The three acts can thus be described as vectors of winning probabilities.

Now consider three possible preferences over these three acts. Preference $\succ_{0}$ is not consistent with the Savage axioms and given by $f_{0} \succ_{0} f_{1} \sim f_{2}$. The other two are consistent with the Savage axioms and given by $f_{1} \succ_{1} f_{0} \succ_{1} f_{2}$ and $f_{2} \succ_{2} f_{0} \succ_{2} f_{1}$. Preference $\succ_{1}$ is induced by a subjective belief that places sufficiently high weight on state $R$, while preference $\succ_{2}$ is induced by a subjective belief that places sufficiently high weight on state $W$.

The question I would now like to address is this: Can I design an experiment that allows me to conclude that the DM has definitely preference $\succ_{0}$ and not $\succ_{1}$ or $\succ_{2}$. My answer will be "No!" I now discuss four possible ways of trying to design experiments with the aim to differentiate between preference $\succ_{0}$ on the on hand and $\succ_{1}$ or $\succ_{2}$ on the other. The first approach is to give the DM a single decision problem. The second approach is to give the DM multiple decision problems and a fixed probability distribution over decision problems. Then the DM is asked to make choices for all these decision problems, before finally an "active" decision problem will be chosen according to the given probability distribution and payment will be made according to the choice of the DM for the active decision problem. The third approach is to again give the DM multiple decision problems. 
Again the DM is asked to make choices for all given decision problems. In this case, however, after the DM made all her choices, every single choice will be independently executed and the DM will be paid out the sum of all payments. The fourth approach, finally, is one that has been discussed extensively by Shmaya and Yariv (2008). I will only discuss it briefly in this section. It is as follows. The DM is told that she will have to make choices from multiple decision problems, but is not yet told what all decision problems are. Her choices will be recorded and ultimately she will be paid either the sum as in the third approach, or the appropriate payment of a randomly chosen active decision problem as in the second approach.

\subsection{A single decision problem}

The DM is now given a single decision problem. There are 4 possible non-trivial decision problems given the three acts. Suppose the DM is given the decision problem to choose from $\left\{f_{0}, f_{1}\right\}$. What can we learn from the DM's potential choices? If she chooses $f_{0}$ the DM could have preference $\succ_{0}$ or $\succ_{2}$. If she chooses $f_{1}$ her preferences could be either $\succ_{0}$ or $\succ_{1}$. No matter what her choice is we cannot conclude that she must have preference $\succ_{0}$. Similarly the decision problems $\left\{f_{0}, f_{2}\right\}$ and $\left\{f_{1}, f_{2}\right\}$ if posed alone cannot be used to deduce that a DM must have preference $\succ_{0}$.

The final and, of course, most interesting decision problem is the one with all three acts $\left\{f_{0}, f_{1}, f_{2}\right\}$. I shall now argue, and this is the crux of this paper, that for any rational DM two things must be true. First, the DM must be allowed to choose an act randomly. In the language of game theory, I consider the three acts given in the decision problem as the DM's pure strategies. Her mixed strategies are all probability distributions over her pure strategies. Given that the DM chooses this probability distribution it is of course objectively known to her. This assumption, always made in game theory, can hardly be controversial.

Second, the DM interprets the effect of objectively randomizing among acts in a pointwise way. This I shall, in the main body of the paper, call the Axiom of Point-Wise Understanding of (Objective) Mixtures over Acts (PUMA). It states that the DM considers every possible state and considers the new objective probability distribution that would result if this were the state. For the given decision problem these two assumptions imply that the DM can actually choose from a bigger set of acts. In fact, the DM can choose from the convex hull of these three acts. Among all the possible mixed strategies, one particularly interesting one, denote it by $\sigma$, is to choose act $f_{1}$ and act $f_{2}$ with equal probability of $\frac{1}{2}$ each. The axiom PUMA then says that the DM must be indifferent between $\sigma$ and the (constant) act which gives her a winning probability of $\frac{1}{2}$ in each of the two states.

In order to help the reader to understand the axiom PUMA better I here offer a discussion of its consequences for a real-life situation with Knightian uncertainty. Your English friend Bertie Wooster offers you a bet on a cricket game between two local cricket teams. You have really no understanding of cricket and have no clue as to which of the two teams is better. You are, however, guaranteed that the game will end in one team 
winning (and the other losing). Bertie, being fair-minded, allows you to choose which team you want to bet on and offers you even odds (of 1:1) in either case. So if you chose to put one pound sterling on team A, you receive two pounds sterling if team A wins (a net win of 1), and nothing if team $B$ wins (a net loss of 1 ). The analogue is true if you decide to put your bet on team B. You can also decline to bet at all, but then you would be considered, by Bertie and many others, as "unsportsmanlike", which is something you would like to avoid, even if perhaps not at all cost.

Now what does Axiom PUMA imply in this setting? Suppose you decide to accept the bet and choose to flip a fair coin to decide on which team you will bet. Then axiom PUMA says that you realize that the outcome of the coin-toss and, thus, your choice, is independent of the state of nature (being the team that will ultimately win). Thus, you consider the probability of team A winning a fixed thing (although unknown), which is certainly not influenced by the outcome of your own independent coin-toss or your ultimate decision.

A second axiom (of Expected Utility for Objective Lotteries (EUOL); usually assumed in the literature) states that the DM uses one and the same expected utility function to evaluate objective lotteries in every state. That is, if she knew the state, she would use this given expected utility function to evaluate the act (that is, the lottery induced by the act in the given state). The axiom EUOL further states that if two acts are such that one provides a higher expected utility than the other in each and every state, then the former act must be strictly preferred over the latter ${ }^{5}$ For the decision problem at hand, allowing the DM to choose acts randomly in conjunction with the two axioms imply that act $f_{0}$ is "strictly dominated" by the random act $\sigma$ and, thus, can never be chosen by the DM (if her preferences satisfy the two axioms).

But this means that any rational DM's realized choices can only be either $f_{1}$ or $f_{2}$. From this we again learn very little, not enough to let us conclude that the DM must have preference $\succ_{0}$ as one of the Savage-consistent preferences $\succ_{1}$ or $\succ_{2}$ is also always consistent with the DM's choice.

In Section 4 in Theorem 11 I show that, for any finite state space, for any outcome space, for any single decision problem and for any preference satisfying Axioms EUOL and PUMA there is a preference that satisfies the Savage axioms that provides the exact same optimal choices for the given decision problem. The exact statement of this theorem as well as its proof is given in Section 4, but a sketch of the proof can be given here.

Any act can be written as a vector of expected utilities, where each coordinate represents one state. In the above example an act was even more simply a vector of winning probabilities, one entry for each of the two states. Fix an arbitrary set of acts, that is fix an arbitrary set of vectors in the appropriate subset of $\mathbb{R}^{k}$, where $k$ is the number of states in $\Omega$. Then the axiom PUMA implies that the actual available sets of acts in vector representation is simply the convex hull of all the given vectors. Suppose we have an act with an expected utility vector for which there is another expected utility vector in this

\footnotetext{
${ }^{5}$ In fact if one accepts that both sources of objective randomness, the DM's personally generated randomness and the objective lotteries paid out in the end, are of the same kind, then Axiom EUOL implies Axiom PUMA.
} 


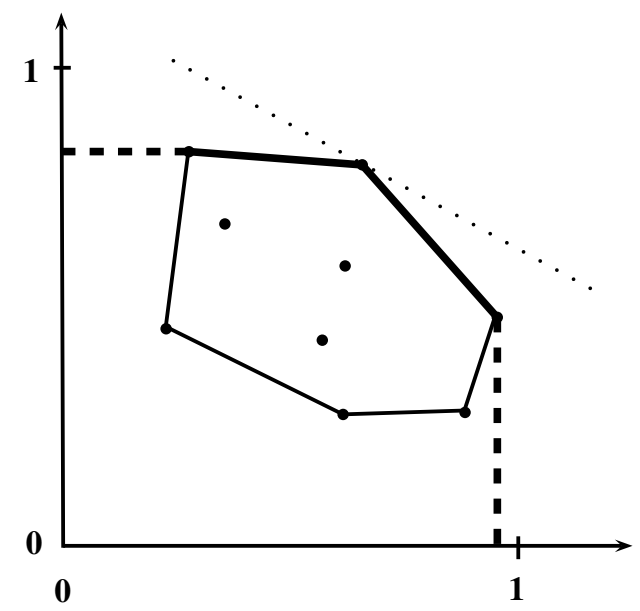

Figure 1: Dots are expected utility (probability of winning) vectors for a given set of acts in the case of two states and outcomes only 0 or 1 . Any expected utility vector not on the solid (or dashed) boundary cannot be chosen by a DM whose preferences satisfy axioms EUOL and PUMA. The dotted line is a separating hyperplane, which yields beliefs that can be used to rationalize the choice of act leading to the expected utility vector that is on the hyperplane.

convex hull to the "upper right" of it. Then there is a possibly random act (by axiom PUMA) such that, by the axiom EUOL, this random acts must be strictly preferred over the original act.

In other words any act that has an expected utility vector in the interior of the above mentioned convex hull cannot be chosen by any DM whose preference satisfies axioms EUOL and PUMA. On the other hand acts with expected utility vectors on the (upperright) boundary of the afore mentioned convex hull can be an optimal for a DM whose preference satisfies the Savage axioms. By the separating hyperplane theorem there is a belief that makes this choice optimal.

Thus, any choice a DM makes, whose preferences satisfy axiom EUOL and PUMA, can also be made by a DM whose preferences satisfy the Savage axioms.

A graphical depiction of the proof of Theorem 1 is given in Figure1. Dots are expected utility (probability of winning) vectors for a given set of acts in the case of two states, $\Omega=\{R, W\}$, and outcome set $Y=\{0,1\}$. The convex hull of all possible expected utility (probability of winning) vectors induced by randomly chosen acts is given by the set bounded by the solid lines. Any expected utility vector not on the thick solid (or thick dashed) boundary cannot be chosen by a DM whose preferences satisfy axioms EUOL and PUMA. The dotted line is a separating hyperplane, which yields beliefs that can be used to rationalize the choice of act leading to the expected utility vector that is on the hyperplane. 


\subsection{A randomly chosen "active" decision problem}

Another attempt to infer a DM's true preferences from her choices can be given as follows. Suppose we do not give the DM one decision problem, but two (or more). For the example the two most promising decision problems are $\left\{f_{0}, f_{1}\right\}$ and $\left\{f_{0}, f_{2}\right\}$. The DM is asked to make choices for both decision problems. Then, one decision problem is chosen according to some fixed objective probability distribution (which the DM is informed about) to be "active", meaning the DM is paid according to her choice for the active decision problem only. For the example, say each of the two decision problem is chosen to be active with equal probability $\frac{1}{2}$.

Now, one might hope that the DM with non-Savage preference $\succ_{0}$ will choose $f_{0}$ in both decision problems, thus differentiating herself from a DM with Savage-preferences $\succ_{1}$ or $\succ_{2}$. If the DM's preference satisfies EUOL and PUMA this, however, is not the case. Note that the DM has now four pure strategies: choose $f_{0}$ or $f_{1}$ for the first decision problem and $f_{0}$ or $f_{2}$ in the second. Note that if the DM chooses $f_{1}$ in the first and $f_{2}$ in the second decision problem, the objective distribution over decision problems, in this case, does the same job the DM's chosen randomization over acts did in the example with a single decision problem. Under axiom PUMA choosing $f_{1}$ in the first and $f_{2}$ in the second decision problem gives the DM another act which has winning probability of $\frac{1}{2}$ in each state, and, thus "strictly dominates" act $f_{0}$.

This means that no DM whose preference satisfies axioms EUOL and PUMA can choose $f_{0}$ in both decision problems. Any other choice, however, is also consistent with one of the two Savage-preferences $\succ_{1}$ or $\succ_{2}$. Thus, as in the case of a single decision problem, this design does not allow us to determine whether a DM's preference, as long as it satisfies axioms EUOL and PUMA, violates the Savage axioms or not.

To see that this point holds in full generality note that this design actually can be seen to be the same as giving the DM a single decision problem. It is as if the DM is simply given a set of 4 random acts to choose from, the DM can even randomize among those if that is useful to her. Thus, Theorem 11, for the single decision problem case, applies also in this case.

\subsection{Multiple decision problems}

My final attempt to infer a DM's true preferences from her choices is as follows. The $\mathrm{DM}$ is, as in the previous subsection, given not one but two or more decision problems. The DM provides a list of choices, one for each decision problem. After that the DM's choices are implemented and the DM is paid the sum of all accruing payments. As in the previous subsection, the two most promising decision problems are $\left\{f_{0}, f_{1}\right\}$ and $\left\{f_{0}, f_{2}\right\}$. The DM is asked to make choices for both decision problems. As before the DM has four pure strategies.

Suppose the DM has a belief that the probability that a red ball is drawn whenever we draw a ball from the urn is equal to some $\mu \in[0,1]$. Then the distribution of payoff in monetary units for each of the four pure strategies and for each belief $\mu$ is as follows 
$\left(\right.$ let $\left.p=\frac{49}{100}\right)$.

\begin{tabular}{c|cccc} 
payoff & $f_{0}, f_{0}$ & $f_{0}, f_{2}$ & $f_{1}, f_{0}$ & $f_{1}, f_{2}$ \\
\hline 2 & $p^{2}$ & $p(1-\mu)$ & $\mu p$ & $\mu(1-\mu)$ \\
1 & $2 p(1-p)$ & $1-p(1-\mu)-(1-p) \mu$ & $1-\mu p-(1-p)(1-\mu)$ & $1-2 \mu(1-\mu)$ \\
0 & $(1-p)^{2}$ & $(1-p) \mu$ & $(1-p)(1-\mu)$ & $(1-\mu) \mu$
\end{tabular}

Consider first the pure strategy $f_{1}, f_{2}$. That is, the DM chooses act $f_{1}$ from the first decision problem and act $f_{2}$ from the second. Note that, in this design, the reduction of compound lotteries is not viable. Consider first the belief that $\mu=\frac{1}{2}$ (this can be thought of as the belief that there is an equal number of 50 red and white balls each in the ambiguous urn). This belief is very different from the belief that $\mu=0$ or $\mu=1$ with equal probability (this can be thought of as the belief that either all balls are red or all balls ar white with equal probability). Given we have two independent draws from the ambiguous urn, the resulting distribution of monetary payoffs are very different for the two different beliefs. In the first case, in which the DM believes that $\mu=\frac{1}{2}$ with certainty, it is possible to win twice. That is, a monetary payoff of 2 is possible. In the second case, in which the DM believes that $\mu=0$ or $\mu=1$ with equal probability, a monetary payoff of 2 is not possible. In fact the two distributions of monetary payoffs for the two beliefs are given as follows.

\begin{tabular}{c|cc} 
payoff & $\mu=\frac{1}{2}$ with prob 1 & $\mu=0$ or $\mu=1$ with prob $\frac{1}{2}$ each \\
\hline 2 & $\frac{1}{4}$ & 0 \\
1 & $\frac{1}{2}$ & 1 \\
0 & $\frac{1}{4}$ & 0
\end{tabular}

If a risk averse DM could choose between beliefs (which, of course, she cannot), she would prefer the second belief. In any case the two beliefs (which would be equivalent for the examples in the previous subsection) are not equivalent here. In fact, this is a point that has been made by Halevy and Feltkamp (2005).

The implications of this "non-viability" of the reduction of compound lotteries, is this. We must allow the DM to hold a "higher order" belief. As it has possible payoff consequences, the DM must be allowed to have a belief that is a probability distribution over the number (or proportion) of red balls in the urn.

This implies that we have to modify not only the payoff space (which has to be adapted from $Y=\{0,1\}$ to $Y^{*}=\{0,1,2\}$ to accommodate all possible sums of monetary payoffs) but also the state space from $\Omega=\{R, W\}$ to $\Omega^{*} \subset \Delta(\Omega)$. I assume that $\Omega^{*}$ can be taken to be any finite subset.

But then, for each new state, say $\mu \in \Omega^{*}$, an act specifies an objective lottery, which the DM, by force of axiom EUOL, evaluates with her given expected utility function. Thus, the problem is again identical to the case of giving the DM a single decision problem with four acts to choose from. Allowing the DM to objectively randomize when choosing acts, and assuming axiom PUMA the main result of this paper, Theorem 1 again applies. 
There is no set of decision problems we can use to differentiate between any preference that satisfies axioms EUOL and PUMA and a Savage preference.

To complete the example, note that, no matter how we choose the state space $\Omega^{*}$ exactly, the pure strategy $f_{0}, f_{0}$ is strictly dominated by the mixture of $\frac{1}{2}\left(f_{0}, f_{2}\right)+\frac{1}{2}\left(f_{1}, f_{0}\right)$. Thus, no DM with preferences satisfying axioms EUOL and PUMA can choose $f_{0}, f_{0}$. All other choices, however, are consistent also with a Savage preference. Thus, if a DM chooses $f_{0}, f_{0}$ she is simply irrational (if the reader buys my argument that axiom PUMA is a requirement of rationality).

\subsection{Possibly state-dependent sequence of decision problems}

I personally do not know whether I have Savage preferences or something like preference $\succ_{0}$. Let us go back to a single decision problem, which here could now be one of these two: $\left\{f_{0}, f_{1}\right\}$ and $\left\{f_{0}, f_{2}\right\}$. Suppose I am asked to choose from the first decision problem. Suppose I have Savage preferences. How should I form my belief about the states? I have only two pieces of information to help me form such a belief. The first piece of information is that there are two colors, red and white. I could believe that somehow red is more prevalent in all things, or more liked by people who fill urns for strange experiments. Of course I could also believe the opposite.

The only other piece of information is the fact that I have been asked to choose from this decision problem and not another. I can have beliefs about the correlation between states and the kind of decision problem I am facing. This does not seem unreasonable to me. In fact I can believe that if the state is red then I am given the second decision problem and when it is white I am given the first one. In some sense I then have preferences $\succ_{0}$, but derived from a Bayesian model, in which I allow beliefs to be not only over states, but also over the sequence of decision problems I am given for the different states.

This criticism of models of ambiguity aversion is not mine. It has been made in perhaps its strongest form by Shmaya and Yariv (2008) to which I refer the reader for a full analysis. See also Al-Najjar and Weinstein (2011).

\section{Setup}

Let $\Omega$ denote the finite set of states. Let $\Delta(\Omega)$ denote the set of all possible (subjective) beliefs over states. Let $Y$ denote the finite set of outcomes. Let $\Delta(Y)$ denote all objective lotteries (all probability distributions over outcomes). Let $f: \Omega \rightarrow \Delta(Y)$ denote an act. Let $\mathcal{F}$ denote the set of all acts. Let $\Delta(\mathcal{F})$ denote the set of objective probability distributions over $\mathcal{F}$.

Let $\succeq$ denote a preference relation on $\Delta(\mathcal{F})$. Let $\sim$ denote its induced indifference relation, and let $\succ$ denote its induced strict preference relation.

Let $u: \Delta(Y) \rightarrow \mathbb{R}^{+}$denote an expected utility function (for objective lotteries). For $f \in \mathcal{F}$ let $u(f)$ denote the vector of the state-wise application of function $u$. That is, $u(f)=\left(u\left(f\left(\omega_{1}\right), \ldots, u\left(f\left(\omega_{k}\right)\right)\right)\right.$ if $\Omega=\left\{\omega_{1}, \ldots, \omega_{k}\right\}$. For $f, g \in \mathcal{F}$ denote $u(f) \gg u(g)$ if 
$u(f(\omega))>u(g(\omega))$ for all $\omega \in \Omega$.

Axiom 1 (Expected Utility on Objective Lotteries (EUOL)) There is a utility function $u: \Delta(Y) \rightarrow \mathbb{R}^{+}$, such that for all $f, g \in \mathcal{F}$ if $u(f) \gg u(g)$ then $f \succ g$.

For $\sigma \in \Delta(\mathcal{F})$ let $\operatorname{supp}(\sigma) \subset \mathcal{F}$ denote the support of $\sigma$. Furthermore, by $s(\sigma)$ denote the state-wise application of the randomization. That is $s(\sigma)(\omega)$ is the objective distribution in state $\omega$ induced by $\sigma$, for all $\omega \in \Omega$.

Axiom 2 (Point-Wise Understanding of (Objective) Mixtures over acts (PUMA)) For any $\sigma \in \Delta(\mathcal{F})$ preference relation $\succeq$ satisfies $\sigma \sim s(\sigma)$.

Thus, if a preference relation satisfies Axiom PUMA then its domain can be taken to be $\mathcal{F}$, as, in this case, we have essentially that $\Delta(\mathcal{F})=\mathcal{F}$. This axiom, thus, states that the objective randomization over acts (to be chosen by the DM) has no influence on the (subjective) likelihood of states $\omega \in \Omega$.

Axiom 3 (Subjective Expected Utility (SEU)) Preference relation $\succeq$ satisfies the Savage Axioms.

\section{A single decision problem}

We shall call $F \subset \mathcal{F}$ a decision problem. We will $F$ also call the DM's set of pure strategies. The DM's set of mixed strategies is given by $\Delta(F)$.

For decision problem $F \subset \mathcal{F}$ and preference $\succeq$ let $c(F, \succeq)=$ $\left\{\sigma \in \Delta(F) \mid \sigma \succeq \sigma^{\prime} \forall \sigma^{\prime} \in \Delta(F)\right\}$. These are the DM's optimal mixed strategies given decision problem $F$ (her mixed best replies to decision problem $F$ ).

Note that, depending on the preference $\succeq$, it is well possible that a pure strategy which has positive weight in an optimal mixed strategy is not itself optimal. In other words, the set of optimal mixed strategies is, in contrast to the case of standard game theory, not necessarily spanned by the optimal pure strategies.

Theorem 1 For any $\succeq$ satisfying Axioms EUOL and PUMA, any decision problem $F \subset$ $\mathcal{F}$, and any $\sigma \in c(F, \succeq)$ there is a preference relation $\succ^{\prime}$ satisfying Axiom SEU such that $\sigma \in c(F, \succeq)$.

Proof: Given $\succeq$ satisfies Axiom EUOL there is an expected utility function $u: \Delta(Y) \rightarrow$ $\mathbb{R}^{+}$which is applied in each state. Let $\mathcal{U}(F)=\{u(f) \mid f \in F\}$ denote the space of expected utility vectors induced by decision problem $F$. Let $\overline{\mathcal{U}}(F)$ denote the convex hull of $\mathcal{U}(F)$. The DM can obtain any expected utility payoff vector in $\overline{\mathcal{U}}(F)$ by choosing the appropriate available mixed strategy $\sigma \in \Delta(F)$.

Let $\sigma \in \Delta(F)$ be a mixed strategy with induced expected utility vector $u^{\sigma}$ with the property that there is a $u^{\prime} \in \overline{\mathcal{U}}(F)$ such that $u^{\prime}(\omega)>u^{\sigma}(\omega)$ for all $\omega \in \Omega$. That is, $u^{\prime}$ strictly dominates $u^{\sigma}$. Then there is a $\sigma^{\prime} \in \Delta(F)$ with induced expected utility vector $u^{\prime}$. But then, as $\succeq$ satisfies Axioms PUMA and EUOL, $\sigma^{\prime} \succ \sigma$. Thus, no DM with 
preferences satisfying Axioms EUOL and PUMA will choose $\sigma$. Now, let $\sigma \in \Delta(F)$ be a mixed strategy with induced expected utility vector $u^{\sigma}$ with the property that $u^{\sigma}$ is on the "top-right" boundary of $\overline{\mathcal{U}}(F)$. That is, there is no $u^{\prime} \in \overline{\mathcal{U}}(F)$ such that $u^{\prime}(\omega)>u^{\sigma}(\omega)$ for all $\omega \in \Omega$. Then, by the separating hyperplane theorem, there is a preference $\succeq^{\prime}$ satisfying Axiom SEU such that $\sigma \in c\left(F, \succeq^{\prime}\right)$.

QED

\section{Randomly chosen active decision problems}

Suppose a DM has to make choices from a set of decision problems $G=\left\{F_{1}, \ldots, F_{n}\right\}$. The $\mathrm{DM}$ is asked to announce her choices for all of these decision problems. After choices are made, the "active" decision problem is chosen according to some a priori known objective probability distribution $\gamma \in \Delta(G)$. The chosen act from the active decision is then used to pay out the DM.

This problem is identical to making one choice from a single decision problem. To see this note that a DM's pure strategies are given by all functions $s: G \rightarrow \mathcal{F}$ with the restriction that $s\left(F_{i}\right) \in F_{i}$. Each pure strategy combined with the objectively known probability distribution over decision problems $\gamma$ is equivalent to an objectively random act $($ a $\sigma \in \Delta(\mathcal{F})$ ). This means that for each pure strategy $s$ there is an act $f \in \mathcal{F}$ such that the DM must be indifferent between pure strategy $s$ and act $f$ if her preference satisfies axiom PUMA. But then the DM's overall decision problem is identical to a single decision problem as studied in Section 4. Thus, Theorem 1 applies, and no such set of decision problems can be used to differentiate the set of preferences that satisfy axioms EUOL and PUMA from the set of preferences that satisfy the Savage axioms.

\section{Multiple decision problems}

Suppose a DM has to make choices from a set of decision problems $G=\left\{F_{1}, \ldots, F_{n}\right\}$. The DM is asked to announce her choices for all of these decision problems. After all choices are made, the DM is paid the sum of all monetary payments resulting from her choices and the realization of randomness. Randomness realizes in an i.i.d. fashion. In the language of urns, a ball is randomly drawn for the first choice, then put back into the urn and another ball is independently drawn for the second choice, and so on. The DM makes all her choices before she learns anything about the composition of the ambiguous urn.

As outcomes are now the sum of multiple possible outcomes, the outcome space has to be adapted. Let $Y^{*}$ denote the appropriate outcome space, derived from the outcome space for single lotteries $Y$. That is, $Y^{*}=\left\{y^{*}=\sum_{i}^{n} y_{i} \mid y_{i} \in Y \forall i\right\}$.

As demonstrated in the example in Section 2.3 for such decision problems the DM must be allowed to have a bigger state space as well. Let this new state space $\Omega^{*} \subset \Delta(\Omega)$ be a finite subset of the set of all probability distributions over $\Omega$.

As in the previous section the DM has pure strategies $s: G \rightarrow \mathcal{F}$ with the restriction that $s\left(F_{i}\right) \in F_{i}$. Given this adapted setup, for any new state $\mu \in \Omega^{*}$, a pure strategy in- 
duces an objective lottery over the new outcome space $Y^{*}$. A pure strategy $s$ is, therefore, simply an act given the new state space and outcome space. Thus, the DM's problem is again identical to a single decision problem with, however, the new state and outcome space. Again, Theorem 1 from Section 4 applies. Again, no such set of decision problems can be used to differentiate the set of preferences that satisfy axioms EUOL and PUMA from the set of preferences that satisfies the Savage axioms.

\section{Discussion}

Another way to view the main result of this paper is this. If an experimenter wants to identify whether a decision maker (DM), under the assumption of Axiom EUOL (expected utility for objective lotteries), has a preference relation which satisfies the Savage axioms or not, then the experimenter needs to give the DM a non-convex set of acts to choose from. However, as the DM can freely and objectively randomize when choosing from the given set of acts, by Axiom EUOL, the DM in fact faces a convex set of acts. Thus, it is not possible to force the DM to make a choice from a non-convex set of acts. Under the assumption of EUOL, the experimenter cannot, therefore, elicit choices that would be inconsistent with Savage preference. Therefore, the experimenter's observations can never be used to test more than the Axiom EUOL.

The experimental study by Halevy (2007) finds that individuals who are uncertainty averse (using the Ellsberg (1961) 2-color urn experiment) typically also fail to reduce compound lotteries and vice versa (using experiments with 2-color urns with different objectively random compositions). In the light of the main result of this paper, this is not surprising, as it demonstrates that any choice that is a violation of the Savage axioms, must be a violation of Axiom EUOL, which of course implies that the DM is able to reduce compound lotteries correctly.

Lo (2000) has shown that in the original Savage (1954) framework, in which outcomes are not necessarily objective lotteries over monetary outcomes, as long as a DM's preference relation satisfies Savage's (1954) Axiom P3 (eventwise monotonicity, as it was termed by Machina and Schmeidler (1992)), her choices can also be rationalized by a preference relation satisfying all of Savage's axioms. This, however, comes at the cost of having to change the DM's preference intensity over outcomes. This is possibly plausible in some cases, but not so much in the case where outcomes are objective lotteries. If outcomes were objective lotteries, Lo's (2000) result would say that for any DM whose preferences satisfy Axiom P3, there is another DM who acts as if she has a single belief over states of nature, but evaluates objective lotteries necessarily quite differently than the first DM. In particular, if the first DM's preferences satisfy EUOL, then the second DM's typically would not. Whether two DM's agree or disagree about how to evaluate objective lotteries, is, however, quite easy to test separately.

My point in this paper is a very different one. If a DM evaluates objective lotteries with a given expected utility function and has otherwise "wild" preferences, then there is another DM who also evaluates objective lotteries with the same expected utility function 
and also satisfies the Savage axioms and who makes exactly the same choice(s), provided we "allow" the DM to choose from a given set of acts randomly.

\section{References}

Al-NajJar, N., And J. L. Weinstein (2011): "The Ambiguity Aversion Literature: A Critical Assessment," Economics and Philosophy, 25, 249-284.

Allais, M. (1953): "Le comportement de l'homme rationnel devant le risque, critique des postulats et axiomes de l'école Américaine," Econometrica, 21, 503-546.

Anscombe, F. J., and R. J. Aumann (1963): "A Definition of Subjective Probability," Annals of Mathematical Statistics, 34, 199-205.

Camerer, C., and M. Weber (1992): "Recent Developments in Modelling Preferences: Uncertainty and Ambiguity," Journal of Risk and Uncertainty, 5(4), 325-370.

EllsBerG, D. (1961): "Risk, ambiguity, and the Savage axioms," The Quarterly Journal of Economics, 75(4), 643-669.

Gilboa, I., And D. Schmeidler (1989): "Maxmin expected utility with non-unique prior," Journal of Mathematical Economics, 18(2), 141-153.

Halevy, Y. (2007): "Ellsberg Revisited: An Experimental Study," Econometrica, 75(2), $503-536$.

Halevy, Y., And V. Feltkamp (2005): "A Bayesian Approach to Uncertainty Aversion," The Review of Economic Studies, 72(2), 449-466.

Klibanoff, P., M. Marinacci, and S. Mukerji (2005): "A smooth model of decision making under ambiguity," Econometrica, 73(6), 1849-1892.

Knight, F. (1921): Risk, Uncertainty and Profits. Boston. MA: Hart, Schaffner \& Marx.

Lo, K. C. (2000): "Rationalizability and the Savage axioms," Economic Theory, 15, $727-733$.

Maccheroni, F., M. Marinacci, and A. Rustichini (2006): "Ambiguity aversion, robustness, and the variational representation of preferences," Econometrica, 74(6), $1447-1498$.

Machina, M., And D. Schmeidler (1992): "A more robust definition of subjective probability," Econometrica, 60(4), 745-780.

RaIffa, H. (1961): "Risk, Ambiguity, and the Savage Axioms: Comment," The Quarterly Journal of Economics, 75(4), 690-694. 
Savage, L. (1954): Foundations of statistics. Wiley.

SCHMEIDLER, D. (1989): "Subjective probability and expected utility without additivity," Econometrica: Journal of the Econometric Society, pp. 571-587.

Shmaya, E., And L. YARIV (2008): "Experiments on Decisions Under Uncertainty: A Theoretical Framework," unpublished manuscript, CalTech.

von Neumann, J., and O. Morgenstern (1944): Theory of Games and Economic Behavior. Princeton University Press. 